\title{
High-order Simulation of Foam Enhanced Oil
} Recovery

\author{
J.M. van der Meer* (Delft University of Technology), D.E.A. van Odyck \\ (Delft University of Technology), P. Wirnsberger (University of Cambridge) \\ \& J.D. Jansen (Delft University of Technology)
}

\section{SUMMARY}

If secondary hydrocarbon recovery methods fail because of the occurrence of gravity override or viscous fingering one can turn to an enhanced oil recovery method like the injection of foam. The generation of foam can be described by a set of partial differential equations with strongly nonlinear functions, which impose challenges for the numerical modeling.

To analyze the effect of foam on viscous fingering, we study the dynamics of a simple foam model based on the Buckley-Leverett equation. Whereas the Buckley-Leverett flux is a smooth function of water saturation, the foam will cause a rapid increase of the flux function over a very small saturation scale. Consequently its derivatives can become extremely large and impose a severe constraint on the time step due to the CFL condition.

Until now, the methods applied to foam EOR processes are only first-order accurate and do not incorporate stabilization near the foam front as far as we know. In order to improve the accuracy near the foam front we make use of total variation diminishing schemes that preserve the numerical stability of the solution. Two dimensional simulations, including gravity, will shed light on the conditions under which foam might exhibit viscous fingering behavior. 


\section{Introduction}

Foam was first applied in the oil industry in the late 1950s to decrease gas mobility and hence reduce the undesirable effect of viscous fingering and gravity override (Fried, 1961). To generate foam in an oil reservoir, usually a mixture of chemicals and water is injected into the reservoir, which together with the injected gas forms a foam. These chemicals make a large contribution to the production costs and therefore the goal is to minimize their amount. To determine the required amount of chemicals for an economically profitable production level, reliable simulations are needed.

There are several models describing foam flow in porous media in use to answer this need. We can roughly distinguish between two classes of foam models: dynamic population balance models, which take into account the strength (bubble density) of the foam, and local equilibrium methods, which incorporate the effect of the foam through a limit function (Ashoori, 2012). The first class of models tries to capture the real dynamics of the process, while the second class assumes that there is only one equilibrium in foam strength. Because of the complex nature of the foam the first class has a higher number of degrees of freedom than the second. So from a computational point of view the second class of models is more suitable for performing large-scale reservoir simulations and therefore most useful for our purpose.

Local equilibrium methods are based on conservation laws, conserving the phases present (usually gas, water and oil), while it is assumed that the surfactant is dissolved in either the gas or the water phase. As soon as the gas comes in contact with a sufficient amount of water and surfactant a foam is generated. The foam will cause a rapid decrease of the gas mobility, because it captures the gas in bubbles that are separated by liquid films (lamellae) between the pore walls (Rossen, 2013). The water mobility is not influenced by foam in these models. Hence the mobility ratio between gas and water is reduced, which will increase the time that the injected gas needs to reach the production well (breakthrough time).

We describe the foam process by an immiscible two-phase flow model where gas is injected in a porous medium filled with a mixture of water and surfactants. The change from pure gas into foam is incorporated in the model through a reduction in the gas mobility. Hence the two-phase flow description of the flow stays intact. Since the total pressure drop in the reservoir is small both fluids can be considered incompressible (Leeftink et al., 2013). As an example we use a two-dimensional quarter five-spot setup, where gas is injected at a constant injection rate via a well at the bottom-left corner and water and gas are produced at the same injection rate via the production well at the right-top corner.

The generation of foam will cause a rapid increase of the flux function over a very small saturation scale. Consequently the derivatives of the flux function can become extremely large and impose a severe stability constraint on the numerical scheme (Rossen, 2013). A first-order upwind scheme might be stable but introduces a lot of numerical diffusion around the shock front. In order to improve the accuracy near the foam front we make use of a higher-order total variation diminishing (TVD) scheme that preserves the numerical stability of the solution. Two-dimensional simulations are then performed to examine the conditions under which foam exhibits viscous fingering behavior and gravity override.

\section{Mathematical model}

\section{State variables and parameters}

The variables involved are the Darcy velocity $\mathbf{u}$, the pressure $p$, the density $\rho$ and the viscosity $\mu$ of the fluids present. Because we deal with a porous medium we express the amount of fluid in terms of the porosity $\phi$ of the medium, defined by the ratio of the pore volume to the total volume, which is assumed constant. The phase saturation $S$, is defined as the volume fraction of the pore space occupied by a fluid phase, so that both phases make up for the total volume. The relative permeability $k_{r}$ depends on specific properties of the fluid and the rock and is defined as a function of saturation. The absolute permeability $k$ depends solely on the properties of the rock and is a function of the spatial variables only. Furthermore $z$ is the depth, $\gamma$ the gravitational acceleration and $\nabla$ denotes the gradient operator. To denote the partial derivative of a variable $q$ in time we write $\frac{\partial q}{\partial t}$. The divergence of a vector $\mathbf{v}$ is denoted 
by $\nabla \cdot \mathbf{v}=\left(\partial_{x}, \partial_{y}\right) \cdot \mathbf{v}$.

\section{Conservation law}

Starting from mass conservation, we can define a basic model for the gas saturation $S_{g}$,

$$
\phi \frac{\partial S_{g}}{\partial t}=-\nabla \cdot\left(f_{g} \mathbf{u}+\lambda_{w} f_{g} \Delta \rho \gamma \nabla z\right)+q_{g},
$$

where $f_{g}$ is the gas fractional flow function, $q_{g}$ the gas flow rate, $\Delta \rho$ the density difference between water and gas, and $\mathbf{u}$ the total Darcy velocity that follows from Darcy's law for the fluid phase velocity,

$$
\mathbf{u}=-k(\lambda \nabla p+\rho \gamma \nabla z)
$$

with the total mobility $\lambda=\lambda(S)$ given by the sum of the phase mobilities

$$
\lambda_{\alpha}=k \frac{k_{r \alpha}\left(S_{\alpha}\right)}{\mu_{\alpha}} .
$$

The fractional flow function is defined as the fraction of the phase mobility and the total mobility. Since the fluids are considered incompressible it follows that

$$
\nabla \cdot \mathbf{u}=q
$$

where $q$ is the total flow rate of water and gas.

\section{Foam model}

If gas comes into contact with a sufficient amount of water and surfactants, foam will form. This will cause a rapid decrease in the gas mobility, which can be modeled by decreasing the relative gas permeability function by a mobility reduction factor $f_{m r}$,

$$
k_{r g}^{f}:=\frac{k_{r g}^{0}}{f_{m r}}, \quad f_{m r}=1+R \cdot F_{w} \cdot F_{s},
$$

where $k_{r g}^{0}$ is the relative gas permeability of the gas in its original state, $R$ is a constant that accounts for the maximum flow resistance of the foam, and $F_{w}$ and $F_{s}$ are functions that describe the sensitivity of the foam to water saturation and surfactant concentration, respectively (Zanganeh et al., 2011). We assume that the surfactant concentration is the same everywhere so that $F_{s}=1$. For $F_{w}$ we use the STARS foam model used in (Leeftink et al., 2013),

$$
F_{w}=0.5+\frac{\arctan \left(\kappa\left(S_{w}-S_{w}^{*}\right)\right)}{\pi},
$$

where $S_{w}^{*}$ is the least amount of water that is needed to form a foam. Since a sudden jump in the mobility of the gas at $S_{w}^{*}$ will cause numerical problems (Rossen, 2013), this jump in mobility is approximated by a continuous arctangent function, so that it is smeared over a width that scales with $1 / \kappa$. In Figure 1 the relative permeability function described here is shown. The flux function and its derivative for the scaled parameters are shown in Figure 2.

\section{Scaling the model}

To reduce the number of parameters we scale the model given by equations (1) and (2) in a similar way as done by Riaz and Tchelepi (2007). If we let $W$ be a characteristic length scale of the model, and $U$ a 


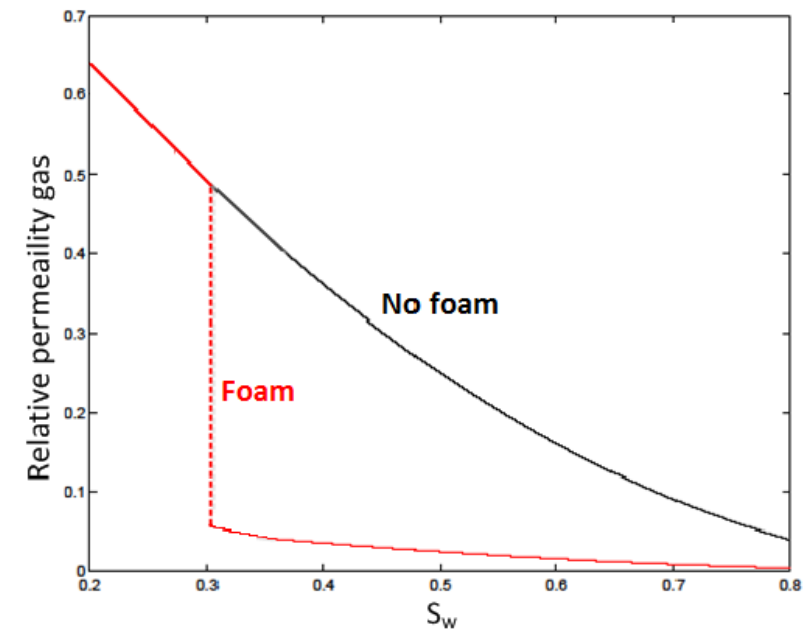

(a) $\kappa=\infty$

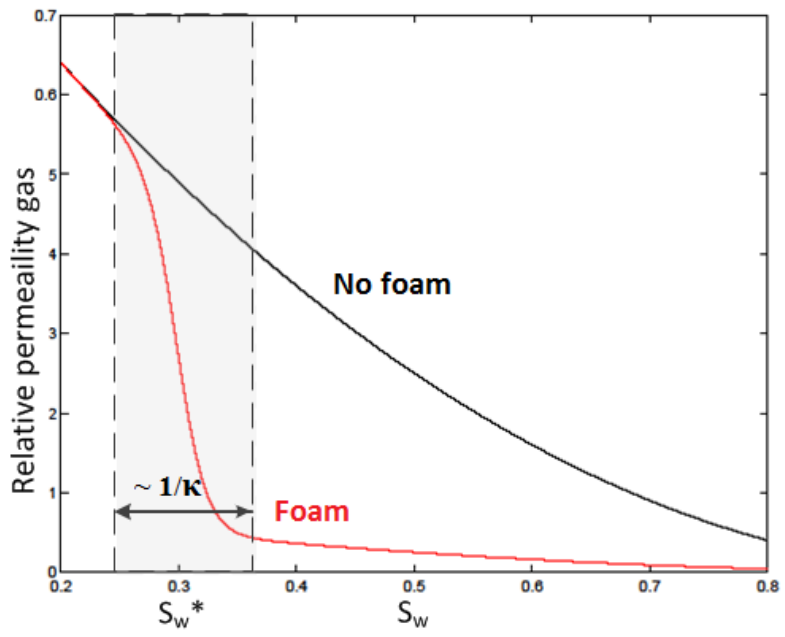

(b) $\kappa=40$

Figure 1 Relative permeability function for a model with and without foam for $S_{w}^{*}=0.3$. The sudden transition due to foam for $R=10$ is approximated by a continuous line at the right with $\kappa=40$.

characteristic velocity scale we can scale the variables as follows

$$
\begin{aligned}
z & =W z^{*}, \\
\nabla & =\frac{\nabla^{*}}{W}, \\
\mathbf{u} & =U \mathbf{u}^{*}, \\
t & =\frac{W}{U} t^{*}, \\
p & =\frac{\mu U W}{k} p^{*}, \\
q & =\frac{U}{W} q^{*},
\end{aligned}
$$

where the superscript ${ }^{*}$ denotes a non-dimensional variable. The relative permeability functions are scaled by their endpoint relative permeabilities, i.e. the value of the relative permeability for the residual water and gas saturation, $S_{w c}$ and $S_{g r}$, respectively. Substituting all of these variables into the dimensional model leads to a non-dimensionalised system of the form,

$$
\begin{aligned}
\phi \frac{\partial S_{g}^{*}}{\partial t^{*}} & =-\nabla^{*} \cdot\left(\frac{k_{r g}^{*} M}{\lambda^{*}} \mathbf{u}^{*}+\frac{k_{r w}^{*} k_{r g}^{*}}{\lambda^{*}} G \nabla^{*} z^{*}\right)+q_{g}^{*}, \\
\mathbf{u}^{*} & =\left(-\lambda^{*} \nabla^{*} \bar{p}+G k_{r g} \nabla^{*} z^{*}\right), \\
\nabla^{*} \cdot \mathbf{u}^{*} & =q^{*},
\end{aligned}
$$

where

$$
\nabla^{*} \bar{p}=\nabla^{*} p_{w}^{*}+\frac{\rho_{w} g k}{\mu_{w} U} \nabla^{*} z^{*},
$$

and $\lambda^{*}=M k_{r g}+k_{r w}$ is the dimensionless mobility function. The variables $M$ and $G$ denote the dimensionless mobility ratio and gravity number respectively, which are given by

$$
\begin{aligned}
M & =\frac{\mu_{w}}{\mu_{g}} \frac{k_{r g}^{*}\left(1-S_{w c}\right)}{k_{r w}^{*}\left(S_{g r}\right)}, \\
G & =\frac{k \Delta \rho g}{\mu_{g} U} .
\end{aligned}
$$




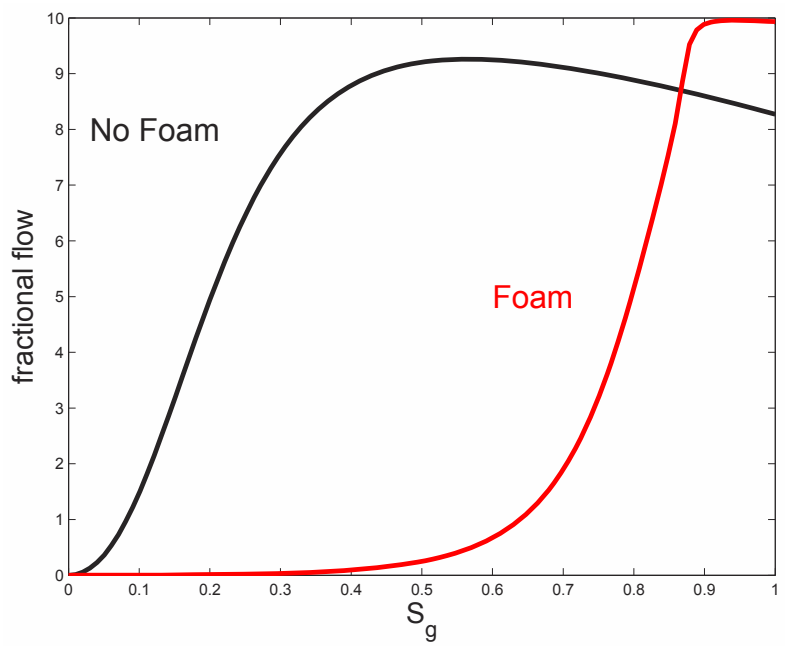

(a) flux

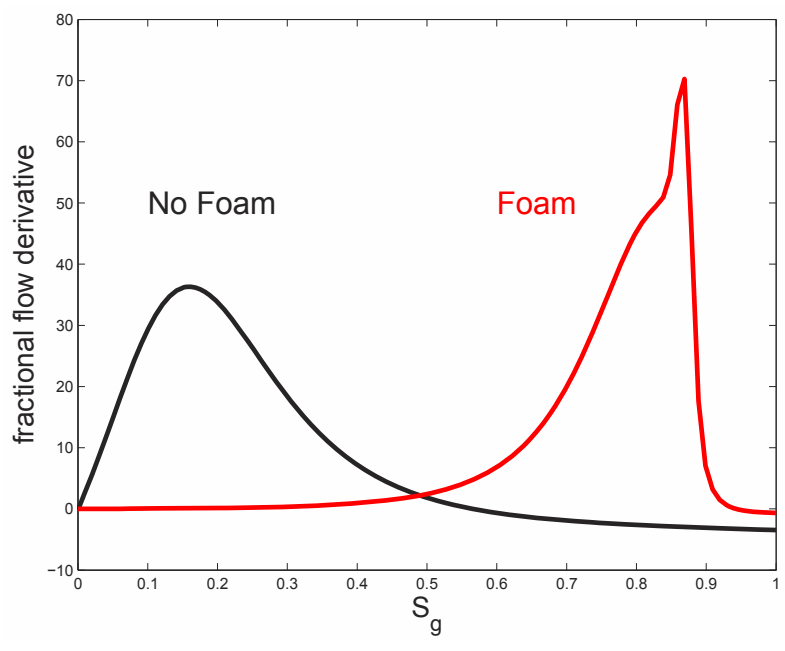

(b) flux derivative

Figure 2 Flux function and derivative for the foam model compared with the Buckley-Leverett flux for $S_{w}^{*}=0.13, \kappa=100, R=1000, M=10$ and $G=2$.

These two variables together with the dimensionless foam parameters $R, \kappa$ and $S_{w}^{*}$, dimensionless injection rate $I^{*}$ and porosity $\phi$, now determine the entire behavior of the fluids for a certain initial boundary value problem. In the rest of the article we will drop the star superscript for readability and define $S$ to be $S_{g}$.

\section{Numerical methods}

The two-phase foam model described in the last section consists of a hyperbolic equation for the saturation (13) and an elliptic equation for the pressure (15). We solve this system by the implicit pressure explicit saturation (IMPES) method, which is designed for this kind of hyperbolic-elliptic problems because it takes into account the different nature of the equations.

We use a structured staggered grid representation of the problem with no-flow boundary conditions everywhere, as shown in Figure 3. The pressure is defined up to an additive constant, so to make sure the system is well-posed the pressure is prescribed in one of the grid cells. The no-flow boundaries are implemented by introducing additional layers of ghost cells around the boundaries. These ghost cells take the same value as the cells inside the boundary so that the net flux across the boundary is zero, i.e. the state variables saturation and pressure defined in the center of a grid cell are equal to the state variables in their corresponding ghost cells. Since we use a staggered grid for the state variables, the Cartesian components of the velocity, $u$ and $v$, are not defined in the cell centers, but at the vertical and horizontal interfaces respectively. To ensure no flow through the boundaries these values are reflected through the boundary. The wells are modeled in the center of each grid cell so that the velocities at the well do not affect the boundary conditions. We represent the wells either as point-sources or as circular wells that are extended over more grid cells.

By using operator splitting, as described in Karlsen et al. (2001), we can split the saturation equation into a hyperbolic conservation law and an ordinary differential equation (ODE) for the mass flow rate. They are solved sequentially using a second-order Strang splitting scheme, which preserves the symmetry of the operator.

\section{Hyperbolic equation}

The hyperbolic equation

$$
\phi \frac{\partial S}{\partial t}=-\nabla \cdot\left(\frac{k_{r g} M}{\lambda} \mathbf{u}+\frac{k_{r w} k_{r g}}{\lambda} G \nabla z\right)
$$




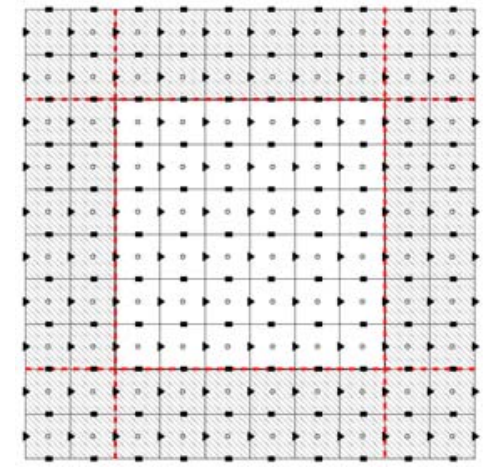

(a) $N x N$ staggered grid

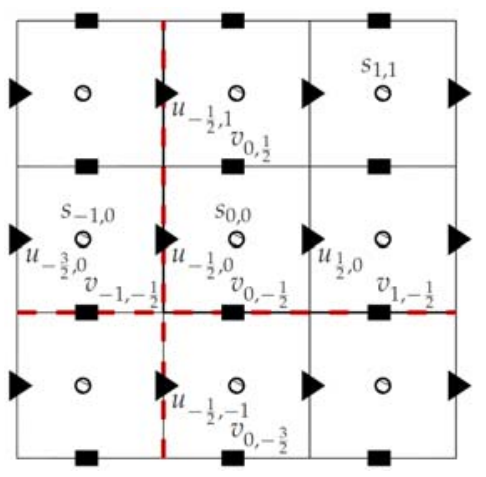

(b) detailed view

Figure 3 Staggered grid with two layers of ghost cells. The red dotted layers represent no-flow boundaries and the black squares and arrows the velocities in the $x$ and $y$ direction, respectively.

is solved with a second order MUSCL scheme, which is a total variation diminishing finite volume method reconstructed from the first order Godunov's method (Toro, 2009). The TVD property prevents instabilities to occur that are due to the numerical method. In other words a TVD scheme is monotonicity preserving if the Courant-Friedrichs-Levy (CFL) condition on the timestep is satisfied (Toro, 2009). Because of the sharp transition in the flux function when foam is generated this condition is very restrictive for the foam model, especially for large values of $\kappa$.

\section{Source term}

The ODE is given by

$$
\phi \frac{\partial S}{\partial t}= \begin{cases}q S_{i n j}, & \text { if } q>0, \\ q S_{g}, & \text { if } q<0 .\end{cases}
$$

In both wells we prescribe the volume flow rate, so that $q=I / V_{\text {well }}$, where $I$ is a constant injection rate and $V_{\text {well }}$ is the volume of the well. The injected fluid saturation $S_{i n j}$ is constant and the produced fluid saturation depends on the saturation present at the production well. The ODE is solved by the second order trapezoidal rule, which is A-stable and therefore suitable for stiff ODEs (Dahlquist, 1963). The size of the timestep of an A-stable method does not suffer from stringent restrictions and the choice only depends on the desired accuracy, but is limited because we cannot exceed the saturation range $0 \leq S \leq 1$. Consequently an additional restriction on the timestep has to be made on top op the CFL condition. We incorporate this by restricting the total variation of the saturation in time for equation (13), so that

$$
\Delta t \leq \frac{\Delta S_{\max } h}{\left(\frac{\partial S^{n+1}}{\partial t}\right)_{\max }} \approx \frac{\Delta S_{\max } h}{\lambda_{\max } \Delta S_{\max }+|q|_{\max } h / \phi},
$$

where $\Delta S_{\max }$ is the maximum variation allowed in the saturation per timestep, $\lambda_{\max }$ is the maximum wavespeed and $h$ is the grid size. Consequently, if the source term is zero everywhere, the maximum timestep boils down to the CFL condition with a CFL number $C_{C F L}=1$. To prevent unstable solutions the maximum variation in the saturation $\Delta S_{\max }$ should be of the order $10^{-3}$ according to Chen et al. (2006). The restriction caused by the source term can therefore cause the timestep to decrease by an significant amount in a foam free setup with $|q|_{\max } h>\lambda_{\max } \Delta S_{\max }$. However, if foam is added, this will cause a strong restriction on the timestep for large $\kappa$ due to the CFL condition, and hence the restriction caused by the source term will be of minor importance. 


\section{Elliptic equation}

For the elliptic equation (15) we use a multigrid linear solver that combines a five-point stencil with a nine-point stencil that is rotated by 45 degrees in order to reduce the grid orientation effect (Wirnsberger, 2012). The five-point stencil accounts for the unrotated coefficients and is combined with a nine-point stencil that accounts for the rotated coefficients and is projected back onto the original grid. The domain is parallelized by MPI using the HYPRE library (Falgout and Meier Yang, 2002) in order to speed up the computations, since roughly $73 \%$ of the simulation time is spent on the pressure solver for this model (Yahya Afiff, 2014).

\section{Results}

We will study the constant injection of pure gas in a water-surfactant filled reservoir for two test cases. In the first case the reservoir is vertical, which causes the gas to flow upwards due to a gravity force and in the second case the reservoir is horizontal and gravity does not play a role. We will take a close look at the behavior of the numerical solutions around the foam front, and compare them to simulation results for the same model without foam (gas-flooding). We use the Brooks-Corey relative permeability functions for gas and water, given by

$$
k_{w r}^{0}=k_{r w e}\left(\frac{S_{w}-S_{w c}}{1-S_{w c}-S_{g r}}\right)^{n_{w}}, k_{r g}^{0}=k_{r g e}\left(\frac{S_{g}-S_{g r}}{1-S_{w c}-S_{g r}}\right)^{n_{g}},
$$

where $k_{r w e}$ and $k_{r g e}$ are the maximum relative permeabilities, $S_{w c}$ and $S_{g r}$ the residual water and gas saturation and $n_{w}$ and $n_{g}$ power coefficients that depend on the rock type. The foam parameters we use are similar to those in the paper of Leeftink et al. (2013) and are listed in Table 1. The dimensionless mobility ratio and gravity number are varied for the different simulations, as well as the grid resolution, error tolerance, end time and the absolute permeability. The other parameters are taken constant (see Table 1).

\section{Case 1: gravity override}

Gravity override occurs when the lighter fluid (in this case gas) separates from the heavier fluid due to gravitational forces. This undesirable event diminishes the sweep area of the reservoir and should therefore be avoided. As shown in Figure 4, the model with foam is less inclined to gravity override than the gas-flooding model. This is caused by the sharp decrease in mobility, so that gravity has a smaller effect on the flux, which follows from the convex-hull construction of the flux as shown in Figure 2(a).

\section{Case 2: viscous fingering}

In order to see viscous fingering a high spatial resolution is needed. We opt for a resolution of 800 grid cells in both directions. Because of the severe CFL constraint on the timestep for the foam model this means we need a timestep that is several orders of magnitude smaller than the timestep for the same simulation without foam. Upon that it takes much longer for the foam front to reach the production well as for the gas front because the front is moving much slower through the reservoir due to the decreased gas mobility. Furthermore we introduce a random heterogeneity in the reservoir to trigger the instabilities so that they show up at an earlier stage of the simulation, to save simulation time. The absolute permeability is generated from a normal distribution with mean $10^{-9}$ and a standard deviation of ten percent. In Figure 5 and Figure 6 the results for these simulations are shown. There is no viscous fingering visible for the foam model, while small fingers appear for the gas-flooding simulation. The hypothesis is that these fingers are suppressed by the sharp transition between foam and water.

In Figure 7 the effect of the foam on the production of water is shown. Since the breakthrough time of the gas front is increased due to foam, the cumulative water production grows over a longer time 


\begin{tabular}{|c|c|c|l|}
\hline parameter & value & dimension & explanation \\
\hline$\alpha$ & $\pi / 2$ & radians & tilting angle \\
$\varepsilon$ & - & - & error tolerance for the linear solver \\
$G$ & - & - & gravity number \\
$k$ & $10^{-9}$ & $\mathrm{~m}^{2}$ & absolute permeability (mean value) \\
$\kappa$ & $3.4 \cdot 10^{4}$ & - & steepness parameter foam transition \\
$I$ & $1.840 \cdot 10^{-4}$ & $\mathrm{~m}^{3} / \mathrm{s}$ & injection relative permeability gas \\
$k_{r g e}$ & 0.8649 & - & endpoint relative permeability gas \\
$k_{r w e}$ & 0.6822 & - & endpoint relative permeability water \\
$L$ & $10^{3}$ & $\mathrm{~m}$ & length of reservoir \\
$M$ & - & - & dimensionless mobility ratio \\
$\mu_{g}$ & $2 \cdot 10^{-5}$ & $P a \cdot g \cdot s$ & gas viscosity \\
$\mu_{w}$ & $10^{-3}$ & $P a \cdot g \cdot s$ & water viscosity \\
$N$ & - & - & resolution of reservoir \\
$n_{g}$ & 2 & - & power coefficient of gas \\
$n_{w}$ & 3.5 & - & power coefficient of water \\
$\phi$ & 0.3 & - & porosity \\
$R$ & $10^{5}$ & - & maximum resistance foam to flow \\
$\rho_{g}$ & 1 & $\mathrm{~kg} / \mathrm{m}^{3}$ & gas density \\
$\rho_{w}$ & $10^{3}$ & $\mathrm{~kg} / \mathrm{m}^{3}$ & water density \\
$S_{g, r}$ & 0.0 & - & residual gas saturation \\
$S_{g, i n j}$ & 1.0 & - & injected gas saturation \\
$S_{w, c}$ & 0.0 & - & critical water saturation \\
$S_{w}^{*}$ & 0.13 & - & critical water saturation \\
$U$ & 1 & $\mathrm{~m} / \mathrm{s}$ & characteristic velocity \\
\hline
\end{tabular}

Table 1 Simulation parameters, a value of - means that the parameter is not constant.

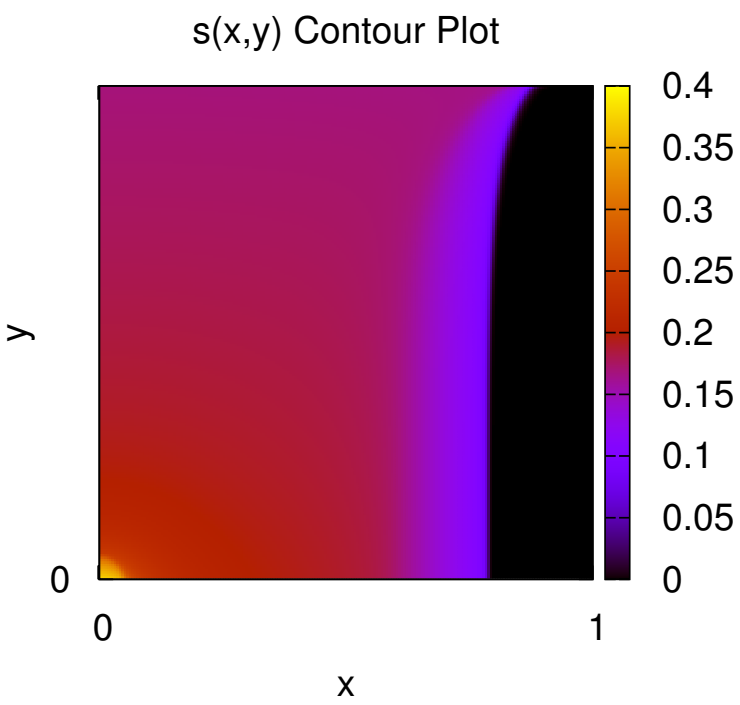

(a) gas-flooding

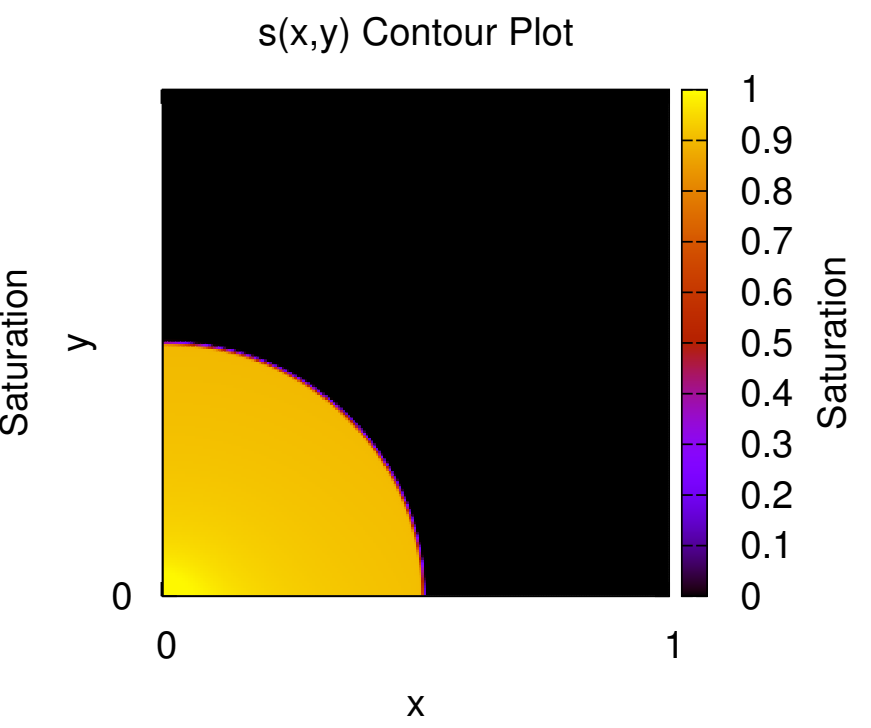

(b) foam EOR

Figure 4 Gas saturation contour plots for a vertical reservoir with $t=100, N=200, M=100, G=2$, $k=10^{-9}$ and $\varepsilon=10^{-6}$. 


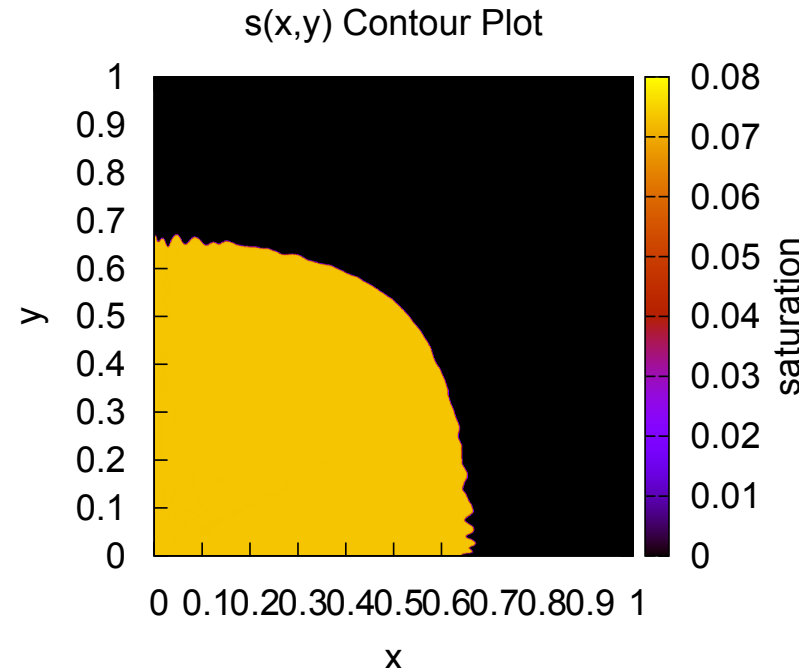

(a) gas-flooding $\mathrm{s}(\mathrm{x}, \mathrm{y})$ Contour Plot

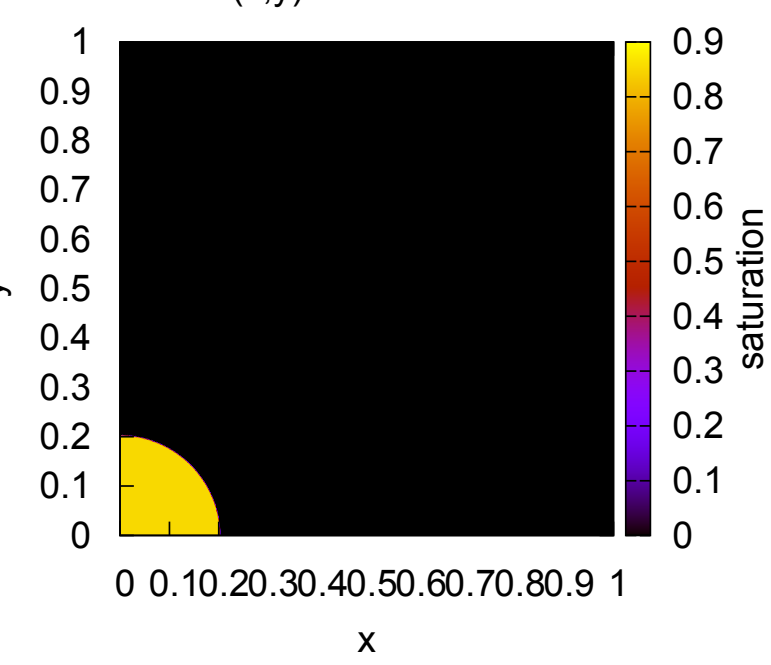

(b) foam EOR

Figure 5 Gas saturation contour plots for a horizontal reservoir with $t=150, N=800, M=50, G=0$, $k_{\text {mean }}=10^{-9}$ and $\varepsilon=10^{-10}$.

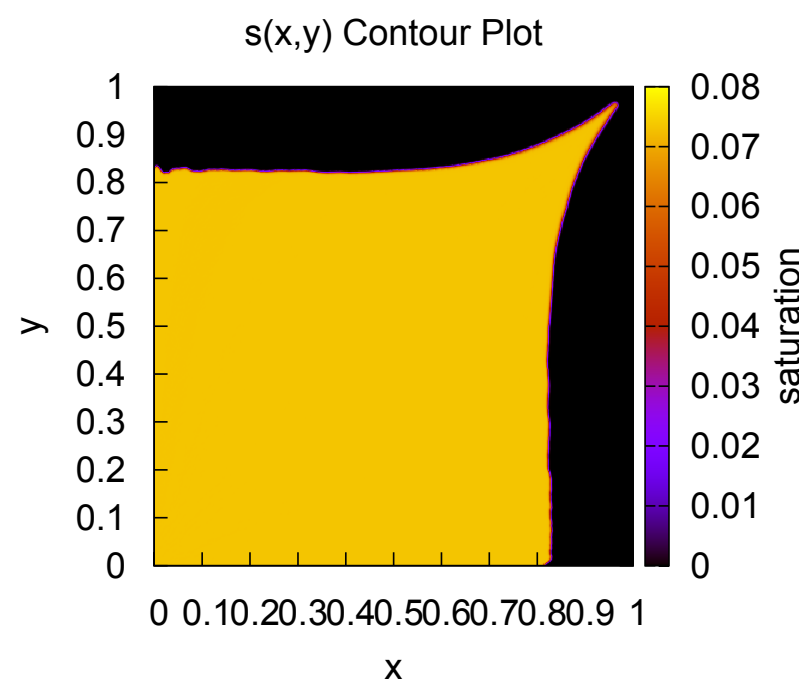

(a) gas-flooding $s(x, y)$ Contour Plot

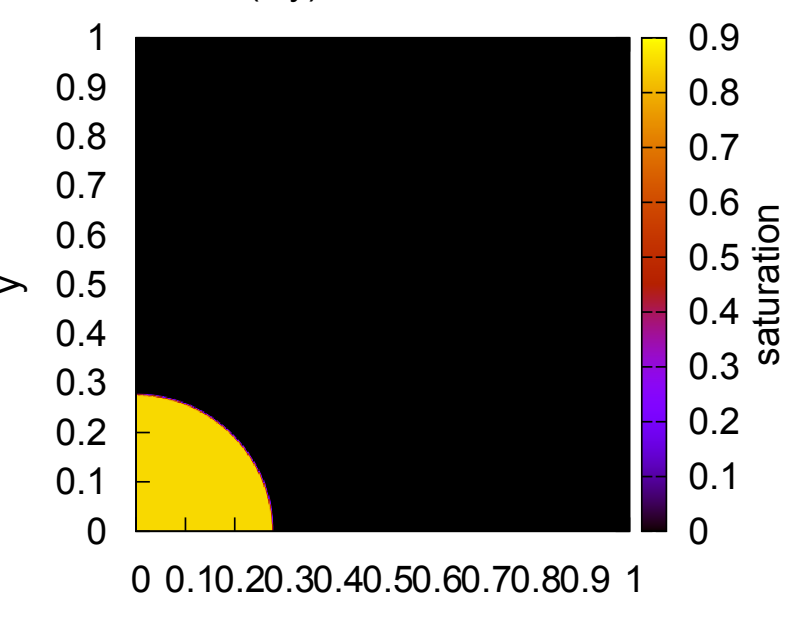

$\mathrm{x}$

(b) foam EOR

Figure 6 Gas saturation contour plots for a horizontal reservoir with $t=280, N=400, M=50, G=0$, $k_{\text {mean }}=10^{-9}$ and $\varepsilon=10^{-10}$. 


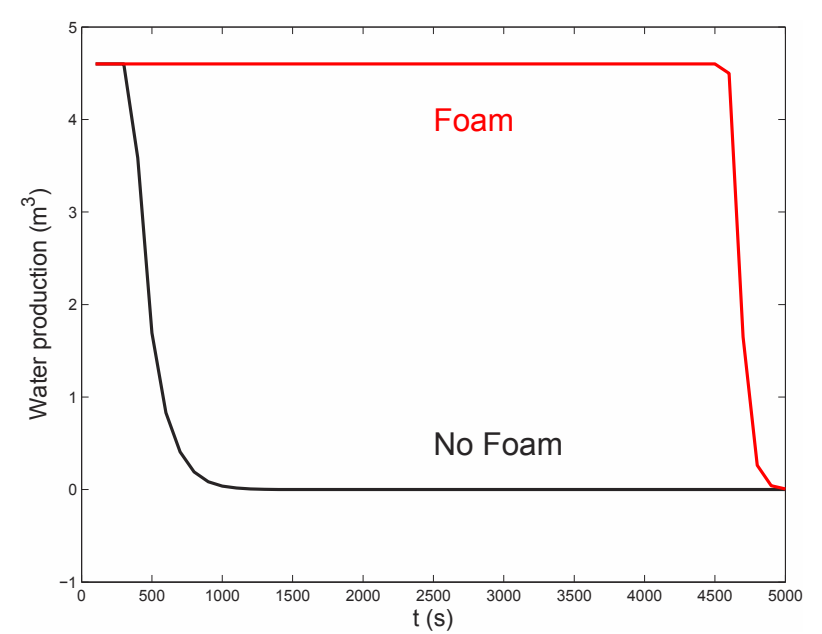

(a) Absolute production

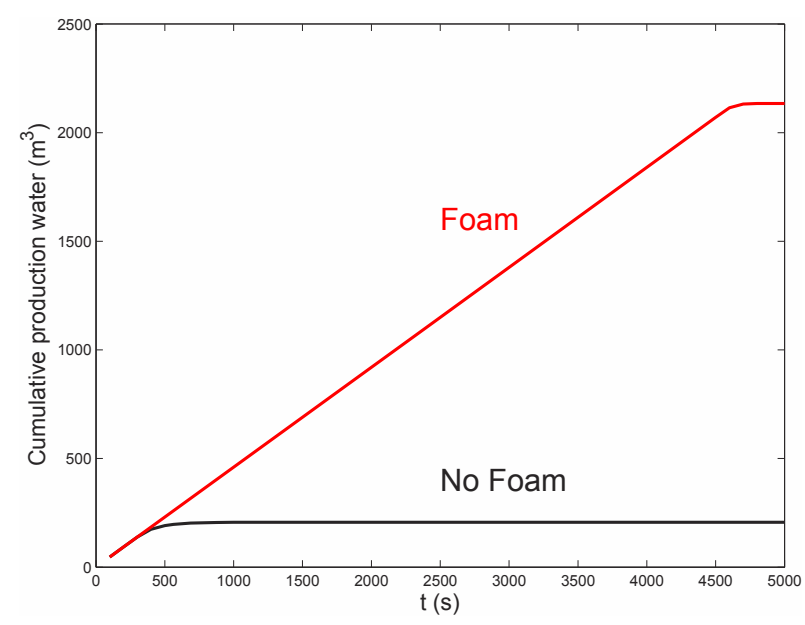

(b) Cumulative production

Figure 7 Water production for a horizontal reservoir with $N=30, M=50, G=0, k_{\text {mean }}=10^{-9}$ and $\varepsilon=10^{-6}$.

range and so the total production is much larger for the foam model. The gas-flooding model is mainly producing gas after the breakthrough time is reached. We note that our model does not contain oil, and that the increased water production is therefore merely an illustration of the positive effect of foam on the gas breakthrough time.

\section{Conclusions}

The results show that foam has an effect on the shock saturation. In comparison with gas-flooding the foam solution has a much lower shock saturation for the water phase and therefore a sharper shock front. By closer examination the shock saturation is almost equal to the critical saturation. This also follows from the convex-hull construction of the flux function with foam.

It turns out that foam has a significant effect on the behavior of the gas front. As expected the simulations with foam suffer less from gravity override and viscous fingering than the simulations without foam. This leads to a better sweep of the reservoir and a much lower breakthrough time. Although these advantages of foam are already well-known in the reservoir engineering community, to our knowledge these phenomena have not yet been studied using higher-order schemes.

Ongoing work includes a quantitative analysis of the instabilities at the gas front with and without foam. In order to do this we need to increase the grid resolution, as well as the order of the numerical methods. To verify our results we plan to compare them with real-world reservoir data.

\section{Acknowledgements}

The first author would like to thank Dr. Nikiforakis for providing the opportunity to visit the Centre for Scientific Computing (Cavendish Laboratory, University of Cambridge) and to the other staff and students at the Centre for Scientific Computing for their help and hospitality. Futhermore we thank Bill Rossen of Delft University of Technology for useful discussions.

\section{References}

Ashoori, E. [2012] Foam for Enhanced Oil Recovery: Modeling and Analytical Solutions. Ph.D. thesis, Delft University of Technology.

Chen, Z., Huan, G. and Ma, Y. [2006] Computational methods for multiphase flows in porous media. SIAM, 1st edn.

Dahlquist, G.G. [1963] A special stability problem for linear multistep methods. BIT Numerical Mathematics, 3 (1), 27-43.

Falgout, R.D. and Meier Yang, U. [2002] hypre: a Library of High Performance Preconditioners. In: Precondi- 
tioners, Lecture Notes in Computer Science. 632--641.

Fried, A.N. [1961] United States Bureau of Mines Bulletin 5866. Tech. rep., Bureau of Mines.

Karlsen, K., Lie, K.A., Natvig, J., Nordhaug, H. and Dahle, H. [2001] Operator Splitting Methods for Systems of Convection-Diffusion Equations: Nonlinear Error Mechanisms and Correction Strategies. Journal of Computational Physics, 173(2), 636-663, ISSN 00219991, doi:10.1006/jcph.2001.6901.

Leeftink, T.N., Latooij, C.A. and Rossen, W.R. [2013] Injectivity Errors in Simulation of Foam EOR. IOR 2013 From Fundamental Science to Deployment, April 2013, EAGE, St. Petersburg, 16-18.

Riaz, A. and Tchelepi, H. [2007] Stability of two-phase vertical flow in homogeneous porous media. Physics of Fluids, 19(7), 072103, ISSN 10706631, doi:10.1063/1.2742975.

Rossen, W.R. [2013] SPE 16624432 Numerical Challenges in Foam Simulation: A Review. SPE Annual Technical Conference and Exhibition held in New Orleans, October, SPE International, New Orleans.

Toro, E.F. [2009] Riemann Solvers and Numerical Methods for Fluid Dynamics. Springer New York, Inc., 3rd edn.

Wirnsberger, P. [2012] The grid orientation effect in miscible displacement. Mphil dissertation, University of Cambridge.

Yahya Afiff, I. [2014] Reservoir simulation of foam flow using Kepler GPU. Msc. thesis, Delft University of Technology.

Zanganeh, M.N., Kam, S.I., LaForce, T.C. and Rossen, W.R. [2011] The Method of Characteristics Applied to Oil Displacement by Foam. SPE journal, (March), 8-23. 